\title{
Crecimiento de recubrimientos sobre fracturas de acero inoxidable 304 por el método Pechini
}

\section{Coating growth on 304 stainless steel fractures by the Pechini method}

\author{
Santiago-Bautista Lourdes \\ Corporación Mexicana de Investigación en Materiales \\ Gerencia de Desarrollo Humano \\ Correo: Isantiago@alumnos-comimsa.mx \\ https://orcid.org/0000-0002-3842-2108 \\ Hernández-García Héctor Manuel \\ Corporación Mexicana de Investigación en Materiales \\ Gerencia de Desarrollo Humano \\ Correo: hmanuelhdz@comimsa.com \\ https://orcid.org/0000-0003-4525-1382 \\ Muñoz-Arroyo Rita \\ Corporación Mexicana de Investigación en Materiales \\ Gerencia de Desarrollo Tecnológico \\ Correo: rita.munoz@alumnos-comimsa.mx \\ https://orcid.org/0000-0002-6247-3319
}

\author{
Granda-Gutiérrez Everardo Efrén \\ Universidad Autónoma del Estado de México \\ Centro Universitario UAEM Atlacomulco \\ Correo: eegrandag@uaemex.mx \\ https://orcid.org/0000-0002-9316-9627 \\ Ibarra-Castro Marcela Nohemí \\ Corporación Mexicana de Investigación en Materiales \\ Gerencia de Desarrollo Humano \\ Correo: marcela.ibarra@alumnos-comimsa.mx \\ https://orcid.org/0000-0003-4066-631X \\ Almanza-Robles José Manuel \\ Centro de Investigación y de Estudios Avanzados del IPN, Saltillo \\ Correo: manuelalmanza@cinvestav.edu.mx \\ https://orcid.org/0000-0003-2781-6336
}

\section{Resumen}

En este estudio se llevó a cabo el crecimiento de recubrimientos ricos en Ni por el método Pechini sobre fracturas de acero inoxidable 304, para sanar superficies de fractura de piezas metálicas. Una serie de pruebas se llevaron a cabo para establecer la temperatura de calcinación por calorimetría de barrido diferencial (DSC) a $1300^{\circ} \mathrm{C}$ por $10^{\circ} \mathrm{C} / \mathrm{min}$ en mezclas de $\left(\mathrm{CH}_{3} \mathrm{COO}\right)_{2} \mathrm{Ni} \bullet 4 \mathrm{H}_{2} \mathrm{O}$ (acetato de níquel tetrahidratado) con $\mathrm{HOC}(\mathrm{COOH})\left(\mathrm{CH}_{2} \mathrm{COOH}\right)_{2} \bullet \mathrm{H}_{2} \mathrm{O}$ (ácido cítrico monohidratado) y precursores: $\mathrm{HOCH} \mathrm{CHO}_{2}$ $\mathrm{HCH}_{2} \mathrm{OH}$ (glicerol) y $\mathrm{HOCH}_{2} \mathrm{CH}_{2} \mathrm{OH}$ (etilenglicol). Los resultados mostraron que el uso de Etilenglicol es mejor que el Glicerol debido a que muestra una estabilidad térmica a menor temperatura para la calcinación. Posteriormente, fracturas cilíndricas de 0.6 x 1 $\mathrm{cm}$ de acero inoxidable 304 se sumergieron en una solución con Etilenglicol y, se gelaron en una mufla a $110^{\circ} \mathrm{C}$ por $24 \mathrm{~h}$. Adicionalmente, las muestras se calcinaron a $660^{\circ} \mathrm{C}$ por $60 \mathrm{~min}$ bajo un flujo de $200 \mathrm{ml} / \mathrm{min}$ de $\mathrm{Ar}$, a velocidad de calentamiento/enfriamiento de $5^{\circ} \mathrm{C} / \mathrm{min}$ para sintetizar las nanopartículas de Ni sobre las fracturas. El crecimiento del recubrimiento sobre las fracturas se llevó a cabo en un flujo de $\operatorname{Ar}$ de $220 \mathrm{ml} / \mathrm{min}$ a $1100^{\circ} \mathrm{C}$ por $60 \mathrm{~min}$ a una velocidad de calentamiento/enfriamiento de $10^{\circ} \mathrm{C} / \mathrm{min}$. Mediante microscopía óptica y de barrido, se observó que el recubrimiento presenta un espesor de hasta $700 \mu \mathrm{m}$ con una serie de precipitados cuboidales mayores a $1 \mu \mathrm{m}$, así como fases laminares ricos en $\mathrm{Ni}$, Fe y $\mathrm{Cr}$.

Descriptores: Metodo Pechini, acero inoxidable, fractura, niquel, nanoparticulas.

\begin{abstract}
This study presents the first results of Ni-rich coating growth by the Pechini method on 304 stainless steels fractures, in order to healing fracture surfaces of metallic parts. A series of tests were performed to establish the calcination temperature by differential scanning calorimetry (DSC) at $1300^{\circ} \mathrm{C}$ using a heating rate of $10^{\circ} \mathrm{C} / \mathrm{min}$ in mixtures of $\left(\mathrm{CH}_{3} \mathrm{COO}\right)_{2} \mathrm{Ni} \bullet 4 \mathrm{H}_{2} \mathrm{O}$ (Nickel Acetate Tetrahydrate) with $\mathrm{HOC}(\mathrm{COOH})\left(\mathrm{CH}_{2} \mathrm{COOH}\right)_{2} \bullet \mathrm{H}_{2} \mathrm{O}$ (Citric Acid Monohydrate) and precursors: $\mathrm{HOCH}_{2} \mathrm{CHOHCH}_{2} \mathrm{OH}(\mathrm{Glycerol})$ and $\mathrm{HOCH}_{2} \mathrm{CH}_{2} \mathrm{OH}$ (Ethyleneglycol). Results indicate that the Ethyleneglycol precursor is better than the Glycol because it shows a thermal stability at lower temperature of calcination. Subsequently, 304 stainless steel cylindrical fractures $(0.6 \mathrm{~cm}$ in diameter, $1 \mathrm{~cm}$ height) were immersed in a solution of Ethyleneglycol and these samples were gelated at $110^{\circ} \mathrm{C}$ for $24 \mathrm{~h}$ in a muffle furnace. Additionally, these samples were calcined at $660^{\circ} \mathrm{C}$ for $60 \mathrm{~min}$ under an Ar flow of $200 \mathrm{ml} / \mathrm{min}$, using a heating/cooling rate of $5^{\circ} \mathrm{C} / \mathrm{min}$ in order to synthesize the nickel nanoparticles on the fractures. Coating growth on the fractures was carried out at an Ar flow of $220 \mathrm{ml} / \mathrm{min}$ at $1100^{\circ} \mathrm{C}$ for $60 \mathrm{~min}$ at a heating/Cooling rate of $10^{\circ} \mathrm{C} / \mathrm{min}$. By optical and scanning microscopy MEB), it was observed that the coating has a thickness of $\sim 700 \mu \mathrm{m}$ with a series of cuboidal precipitates larger than $1 \mu \mathrm{m}$, as well as laminar phases rich in $\mathrm{Ni}$, Fe and $\mathrm{Cr}$.
\end{abstract}

Keywords: Pechini method, stainless steel, fracture, nickel, nanoparticles. 


\section{INTRODUCCIÓN}

El método Pechini se utiliza por medio de la vía húmeda y posee varias ventajas sobre otros métodos como el mecánico o el químico debido a que es un método simple, de bajo costo y no requiere de equipo sofisticado. Por otro lado, permite obtener materiales con excelente homogeneidad, alta pureza y control sobre el tamaño de nanopartícula a bajas temperaturas $\left(300\right.$ a $\left.600^{\circ} \mathrm{C}\right)$. Este método está clasificado en los métodos de fabricación de la ruta sol-gel (Zaki et al., 2012; Galceran et al., 2007; Rao et al., 2004; Raba et al., 2015; Mota et al., 2013; Xiaoming et al., 2015).

Básicamente, el método Pechini requiere de un precursor de resina polimérica que consiste en una mezcla de cationes formados por un agente orgánico complejo: ácido cítrico o ácido etilendiaminotetraacético (EDTA), así como de una solución de etilenglicol. En este caso, los cationes se transforman en un quelato y se forma una resina polimérica que se descompone a baja temperatura $\left(300^{\circ} \mathrm{C}\right)$. Durante esta transformación se generan dos reacciones: la primera es una formación de complejos de ácido cítrico o ácido etilendiaminotetraacético con iones metálicos y la segunda, una esterificación entre estos ácidos y el etilenglicol, reduciendo cualquier tipo de segregación de los cationes (Pechini, 1967; Vaqueiro y López, 1968). La posterior calcinación de este precursor permite la obtención de nanopartículas de adecuada composición química, a baja temperatura (Raba et al., 2015; Mota et al.,2013; Solarte et al., 2015).

Las propiedades mecánicas de los materiales son diferentes cuando el tamaño de estos disminuye a tamaños nanométricos, estos cambios ocurren inclusive a nivel atómico y molecular. Estas propiedades cambian dependiendo de la forma, tamaño, estabilidad energética y estructura (Johnston, 2002; Puente et al., 2012; Niederberger, 2007; Raba, et al., 2015; Fabian et al., 2015). Las nanopartículas de níquel pertenecen al grupo de los metales de transición y presentan un orbital d parcialmente lleno de electrones, esta característica permite la posibilidad de una hibridación con algún otro elemento químico con el mismo nivel de energía (Blackman, 2009).

Por otro lado, a nivel industrial el desgaste o fractura de la superficie se traduce en la disminución de las propiedades tribológicas y susceptibilidad a la corrosión. Por lo tanto, se recurre a procesos de deposición de recubrimientos metálicos o compósitos de alta resistencia a la corrosión y al desgaste, así mismo que sirvan como substrato para fijar materiales avanzados: grafeno u óxidos de grafeno (Marulanda y Trujillo, 2007; Wang y Bierwagen, 2009, Sahoo y Masanta, 2017; Chen et al., 2016).
En este trabajo de investigación de tipo exploratorio se sintetizan nanopartículas de $\mathrm{Ni} / \mathrm{NiO}$ sobre fracturas de acero inoxidable 304 y, con la aplicación de un tratamiento térmico se generó un recubrimiento rico en níquel que se utilizará en estudios posteriores como sustrato de crecimiento de recubrimientos de ultra-alta dureza.

\section{DesarRollo eXPerimental}

\section{ELECCIÓN DEL PRECURSOR POR DSC PARA SINTETIZAR NANOPARTÍCULAS DE NI}

En la síntesis de las nanopartículas de $\mathrm{Ni}$, se emplearon dos precursores químicos: etilenglicol y glicerol. Con el fin de establecer y seleccionar las temperaturas más bajas para sintetizarlas por el método Pechini. En ambos casos del uso de los precursores, se consideró la relación molar ácido cítrico/etilenglicol y ácido cítrico/glicerol: 1/4. Por separado, ambas relaciones se prepararon en $20 \mathrm{ml}$ de agua desionizada a temperatura ambiente $y$, se agitaron en una parrilla magnética hasta disolver los sólidos. La mezcla se aforó a $60 \mathrm{ml}$ y se calentó sin agitación magnética a $80^{\circ} \mathrm{C}$ para evaporar la solución y reducirla hasta 20 $\mathrm{ml}$ (Puente et al., 2012). Ambas soluciones se introdujeron en una mufla a $110^{\circ} \mathrm{C}$ por $24 \mathrm{~h}$ para obtener el gel. Posteriormente, las muestras de gel se evaluaron por separado en un equipo de calorimetría de barrido diferencial (DSC, por sus siglas en inglés) marca TA Instruments modelo Q600 empleando rampas de calentamiento $10^{\circ} \mathrm{C} /$ min de 25 a $1300^{\circ} \mathrm{C}$ para establecer la temperatura y pérdida de masa por calcinación.

\section{PreparaCión Y CARACTERIZACIÓN DE LAS FRACTURAS DE} LOS ACEROS INOXIDABLES 304

Las fracturas se obtuvieron a partir de barras de acero inoxidable 304 de $0.6 \mathrm{~cm}$ de diámetro y $12 \mathrm{~cm}$ de longitud. Se realizó una muesca al centro de cada probeta y se sometió a una prueba de doblez hasta fracturarlas, empleando una máquina universal Tinius Olsen con capacidad de $60 \mathrm{t}$. Las superficies de fractura se inspeccionaron y analizaron en un microscopio óptico marca OLYMPUS modelo PMG-3 a 50 X y 100X, así como en un microscopio electrónico de barrido (MEB) marca JEOL modelo JSM-6490LV, también se analizó la composición química elemental mediante energía dispersiva de rayos $\mathrm{X}(\mathrm{EDX})$. 
INMERSIÓN DE LAS FRACTURAS EN LA SOLUCIÓN Y POSTERIOR GELACIÓN

Las fracturas se colocaron en un vaso de precipitado de $50 \mathrm{ml}$ con etanol grado industrial para limpieza superficial por 15 min en un equipo de ultrasonido marca Power-Sonic410 modelo LUC-410. Las muestras se secaron bajo un flujo de aire caliente. Posteriormente, se preparó la solución rica en $\mathrm{Ni}$ de acuerdo con el procedimiento experimental descrito en la sección de Elección del precursor por DSC para sintetizar nanopartículas de $\mathrm{Ni}$ y, sumergieron las fracturas y sonicaron por $15 \mathrm{~min}$, con el fin de penetrar la solución entre las microfisuras y microporos. Finalmente, las muestras se introdujeron en una estufa marca ThermoScientific a $110^{\circ} \mathrm{C}$ por $24 \mathrm{~h}$ para obtener la gelación en la superficie de la fractura.

\section{CalCinaCión y CRECIMIENTO DEL RECUBRIMIENTO RICO EN NI}

Las fracturas sumergidas en la solución se calcinaron en un horno tubular marca Thermolyne modelo 59300 con un flujo de Ar de $200 \mathrm{ml} / \mathrm{min}$ a una temperatura de $660^{\circ} \mathrm{C}$ por $1 \mathrm{~h}$, con una velocidad de calentamiento y enfriamiento de $5^{\circ} \mathrm{C} / \mathrm{min}$. Las muestras obtenidas se inspeccionaron y analizaron químicamente en un microscopio electrónico de barrido (MEB) y espectroscopia de energía dispersiva de rayos- $X$ marca JEOL JSM 6490LV/EDAX. Finalmente, se realizó una corrida en MINITAB de un diseño de experimentos $2^{3}$ con valores obtenidos previamente, con la finalidad de conocer las interacciones de la temperatura, tiempo y velocidad de calentamiento respecto al crecimiento del recubrimiento rico en Ni. La Tabla 1 resume los factores y los valores de los mismos. El crecimiento del recubrimiento sobre las superficies de fractura se llevó a cabo en un horno tubular Thermolyne modelo 59300 con un flujo de $\operatorname{Ar}$ de $220 \mathrm{ml} / \mathrm{min}$. Las muestras se inspeccionaron por MEB y EDX para inspeccionar fases e identificar las especies químicas en el recubrimiento.

Previo a la caracterización, se prepararon metalográficamente la parte frontal y transversal de las muestras con recubrimiento y se realizó revelado microestructural electroquímicamente mediante una solución preparada con $40 \mathrm{ml}$ de ácido nítrico $\left(\mathrm{HNO}_{3}\right), 50 \mathrm{ml}$ de ácido sulfúrico $\left(\mathrm{H}_{2} \mathrm{SO}_{4}\right)$ y $10 \mathrm{ml}$ de ácido fosfórico $\left(\mathrm{H}_{3} \mathrm{PO}_{4}\right)$ con una corriente de 2 Volts, 3 Amperes por 3s. Los recubrimientos se inspeccionaron en un estereoscopio marca MOTIC Trinocular modelo SMZ-143 y la caracterización microestructural e identificación especies químicas por MEB/EDX, respectivamente.

\section{DisCUSIÓN Y ANÁLISIS DE RESULTADOS}

\section{SELECCIÓn DEL PRECURSOR PARA EL CRECIMIENTO DE RECUBRIMIENTO}

Las Figuras 1a y b muestran dos termogramas de calcinación por análisis térmico diferencial de barrido y termogravimétrico (ATD-TG) empleando etilenglicol y glicerol, respectivamente. Se aprecia en la figura 1a la pérdida de masa de $37 \%$ y se estabiliza hasta los $\sim 600^{\circ} \mathrm{C}$. Por otro lado, se observan dos eventos térmi$\cos$ a 180 y $270^{\circ} \mathrm{C}$ que indican la pérdida de agua y material orgánico. A partir de los $\sim 450^{\circ} \mathrm{C}$ se postula la formación de níquel metálico. No obstante, en la figura 1 b se observa una pérdida de masa de $35 \%$ y continúa perdiendo masa hasta $\sim 900^{\circ} \mathrm{C}$. Los eventos térmicos bien definidos se aprecian entre 100 y $270^{\circ} \mathrm{C}$, aproximadamente. Las demás fluctuaciones térmicas se deben a las condiciones operativas del equipo. Sin embargo, a $450^{\circ} \mathrm{C}$ se inicia el proceso de obtención del níquel metálico; sin embargo, presenta una estabilización hasta $900^{\circ} \mathrm{C}$ si se analiza a través de la curva TG. Con base en lo anterior, se establece que a la temperatura de $450^{\circ} \mathrm{C}$ es factible obtener níquel metálico, asimismo este hecho se confirma en la literatura (Puente et al., 2012). Comparativamente, a pesar de que la diferencia de la pérdida de masa entre ambos precursores es de $2 \%$, se seleccionó el $\mathrm{HOCH}_{2} \mathrm{CHOHCH}_{2} \mathrm{OH}$ (etilenglicol) para calcinar y crecer recubrimientos ricos en níquel sobre fracturas en aceros inoxidables 304, ya que a temperaturas de $\sim 450^{\circ} \mathrm{C}$ se forma el Ni metálico y se estabiliza a $\sim 600^{\circ} \mathrm{C}$. En contraste, con el glicerol se requiere más temperatura de estabilización $\left(\sim 900^{\circ} \mathrm{C}\right)$.

Tabla 1. Factores utilizados para la ejecución de las pruebas experimentales en el diseño de experimentos $2^{3}$

\begin{tabular}{ccccc}
\hline Factor & Nombre & Tipo & Bajo & Alto \\
\hline A & Temperatura $\left({ }^{\circ} \mathrm{C}\right)$ & Numérico & 1000 & 1200 \\
B & Tiempo $(\min )$ & Numérico & 30 & 60 \\
C & Vel. de calentamiento $(\% \mathrm{~min})$ & Numérico & 5 & 10 \\
\hline
\end{tabular}


INSPECCIÓN DE LAS FRACTURAS PREVIAS AL CRECIMIENTO DEL RECUBRIMIENTO

En la Figura 2 se observa la topografía de la fractura y las mediciones en el espaciamiento de la microgieta. Se aprecia por microscopía electrónica de barrido una superficie con microporos y microgrietas. Las microgrietas tienen anchos de 185 a $293 \mu \mathrm{m}$. Se resalta que la variedad de tamaños de los microporos alcanzan dimensiones menores a $1 \mu \mathrm{m}$ y, en el interior se aprecian partículas ovaladas de naturaleza química elemental de $\mathrm{Mn}, \mathrm{S}$ y Fe que deduce el MnS, principalmente (Figura $2 b$ ). Cabe mencionar que la solución preparada por el método Pechini se introduzce en los microporos y microgrietas y, en la tapa del tratamiento térmico se sellan y crece un recubrimiento metálico rico en níquel sobre la superficie de fractura.

\section{CARACTERIZACIÓN DE LAS FRACTURAS CON GELACIÓN}

Y CALCINADAS PREVIO AL CRECIMIENTO DEL RECUBRIMIENTO

En la Figura 3 se muestran las fracturas de acero inoxidable 304 después de la gelación. Se sumergieron en la solución preparada por el método Pechini utilizando como precursor químico el Etilenglicol. La penetración de la solución en las microgrietas asegura que durante el proceso de calcinación se lleve a cabo el crecimiento de las nanopartículas de níquel (Ni) y, cubre por completo estas discontinuidades durante el crecimiento del recubrimiento por tratamiento térmico.

Las Figuras 4a y b muestran una zona de la topografía y el análisis puntual químico de la cubierta de gel calcinado a $450^{\circ} \mathrm{C}$ por $1 \mathrm{~h}$. Los microporos están totalmente cubiertos por el material calcinado rico en elementos de $\mathrm{Ni}$ y, en menor proporción de $\mathrm{O}$ y Fe. Se considera que cierta cantidad de níquel se oxida a $1000^{\circ} \mathrm{C}$ debido a la presencia de $\mathrm{O}$ en la solución previa a la calcinación. La factibilidad de la formación de nanopartículas $\mathrm{Ni} / \mathrm{NiO}$ en el rango de $450-660^{\circ} \mathrm{C}$ es confirmado en la literatura (Puente et al., 2012). Estos tamaños nanométricos modifican las propiedades físicas del material que permiten una alta reactividad química con el substrato.

La Figura 5a, b y c muestran a altas amplificaciones otra zona por MEB de la fractura calcinada a $450^{\circ} \mathrm{C}$ por 60 min bajo un flujo Ar. La superficie de la fractura frontal muestra que los microporos están completamente cubiertos por material calcinado (Figura 5a). Por otro lado, se aprecia por mapeo en el interior de la microfisura la composición química elemental rico en Ni y $\mathrm{C}$ (Figura $5 \mathrm{~b}$ y c). El contenido de $\mathrm{C}$ se forma durante la etapa de calcinación debido a que el material empleado por el método Pechini es de origen orgánico.
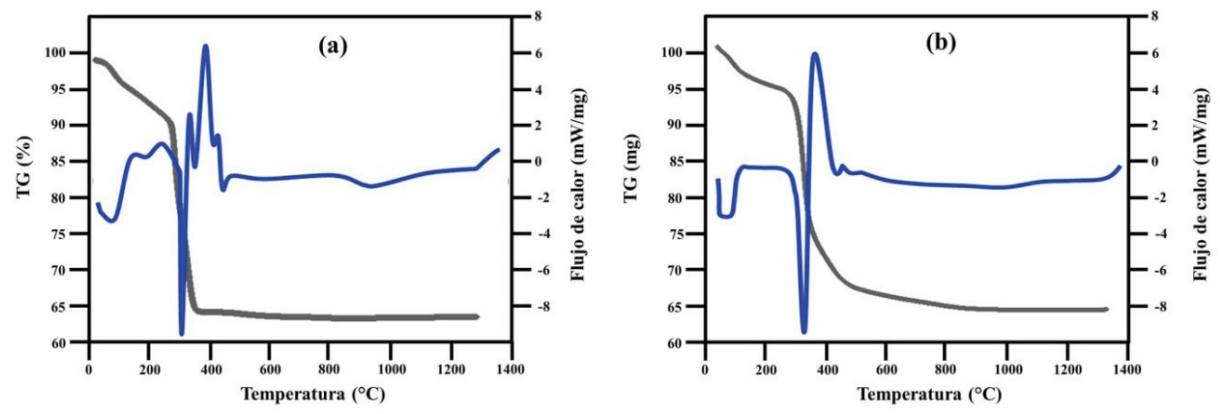

Figura 1. Termogramas Termogravimétricos (TG) y calorimetría diferencial de barrido (DSC) a $1300^{\circ} \mathrm{C}$ de la etapa de calcinación empleando: a) etilenglicol y b) glicerol
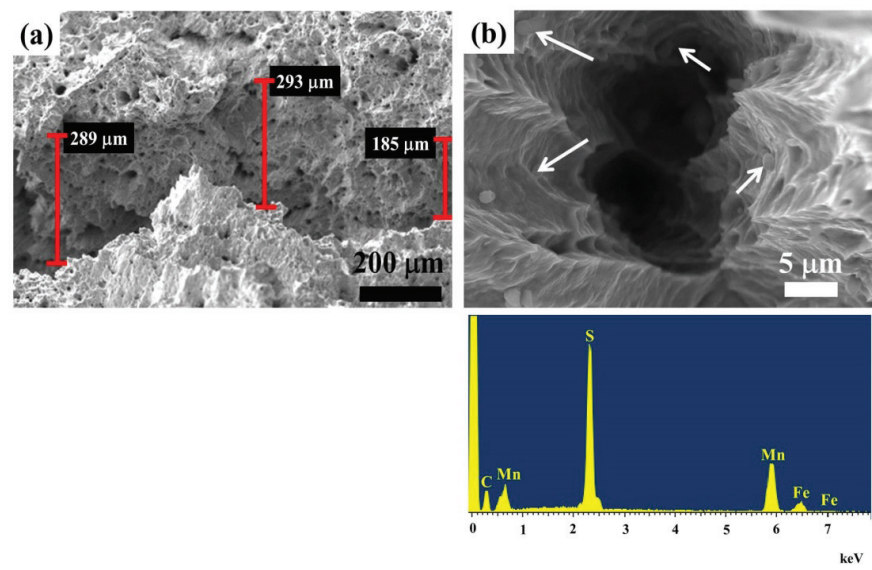

Figura 2. a) Imagen de electrones secundarios de la superficie de fractura de acero inoxidable 304, b) imagen de electrones secundarios y EDX de partículas de $\mathrm{MnS}$ en el interior del microporo 

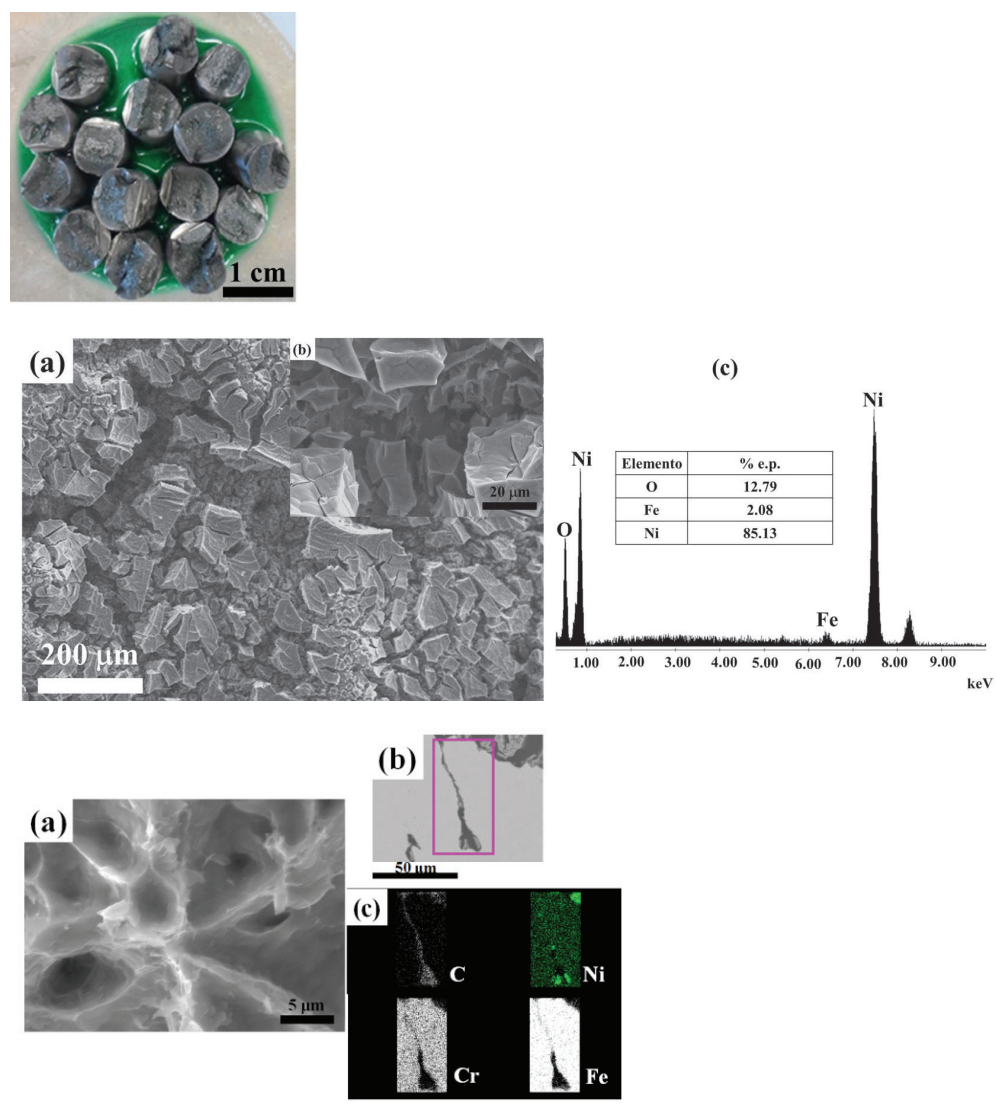

\section{CARACTERIZACIÓN DEL RECUBRIMIENTO SObRE LAS FRACTURAS DE ACERO INOXIDABLE 304}

Para la selección de las muestras a caracterizar se realizó una inspección visual sobre las 16 muestras obtenidas de acuerdo con el diseño de experimentos. El criterio de selección se basó en la presencia o no, de recubrimiento sobre la superficie de fractura. La Tabla 2 resume la identificación de las muestras y las condiciones a las cuales se llevó a cabo la experimentación. Estas variables de entrada se tomaron de la secuencia de ejecución del diseño de experimentos $2^{3}$ realizado.

Cabe señalar que el recubrimiento de las muestras se caracterizó estereoscópicamente para elegir 2 muestras representativas de la serie de experimentos de
Figura 3. Superficies de fractura cubiertas de gel
Figura 4. a) Imagen de electrones secundarios de material calcinado a $450^{\circ} \mathrm{C}$ por $1 \mathrm{~h}$ sobre la fractura de un acero inoxidable 304 , b) espectrograma por EDX de la cubierta calcinada
Figura 5. a) Imagen de MEB de los microporos cubiertos con material calcinado a $550^{\circ} \mathrm{C}$ por $60 \mathrm{~min}$, b) corte transversal de la fractura con material calcinado en el interior de la microfisura, c) mapeo de composición química por EDX

Tabla 2. Identificación de las muestras y condiciones experimentales para el crecimiento del recubrimiento sobre superficies de fractura de acero inoxidable 304

\begin{tabular}{cccc}
\hline Muestra & Temperatura $\left({ }^{\circ} \mathrm{C}\right)$ & Tiempo $(\mathrm{min})$ & Velocidad de calentamiento $\left({ }^{\circ} \mathrm{C} / \mathrm{min}\right)$ \\
\hline A & 1000 & 30 & 10 \\
B & 1000 & 60 & 5 \\
C & 1200 & 30 & 10 \\
D & 1200 & 60 & 10 \\
\hline
\end{tabular}


La Figura 7a muestra la imagen del crecimiento incompleto del recubrimiento sobre la superficie de fractura de la muestra A tratada térmicamente a $1000^{\circ} \mathrm{C}$ por 30 min empleando una velocidad de calentamiento de $10^{\circ} \mathrm{C} / \mathrm{min}$. Se aprecian zonas blancas del recubrimiento incompleto y zonas de color grisáceo mediante la técnica de electrones dispersados en el microscopio electrónico de barrido. Estas formaciones de compuestos se favorecen en las crestas de los microporos debido a una mayor energía superficial. Los espectrogramas de la Figura $7 \mathrm{~b}$ y c muestran la naturaleza química de la zona grisácea (1) y blanca (2) analizada por la técnica de energía dispersiva de rayos- $X$. La Figura $7 b$ muestra la composición química de las zonas grisáceas con picos intensos de níquel $(\mathrm{Ni})$ y picos menos intensos de oxigeno $(\mathrm{O})$, hierro $(\mathrm{Fe})$ y cromo $(\mathrm{Cr})$, así como picos de menor intensidad de Mn. Mientras tanto, las zonas en tonalidad blanca (2) muestran un pico intenso de oxígeno $(\mathrm{O})$ y cromo $(\mathrm{Cr})$, además de los elementos propios de la aleación (Figura 7 c). Se establece que corresponden a óxidos de cromo.

Posteriormente, se realizó el mismo procedimiento para la muestra $\mathrm{B}$ a una temperatura de $1000^{\circ} \mathrm{C}$ por 60 min, empleando una velocidad de calentamiento de $5^{\circ} \mathrm{C} / \mathrm{min}$. En la Figura 8a se muestra sobre el recubrimiento una distribución de partículas de tamaños ma-

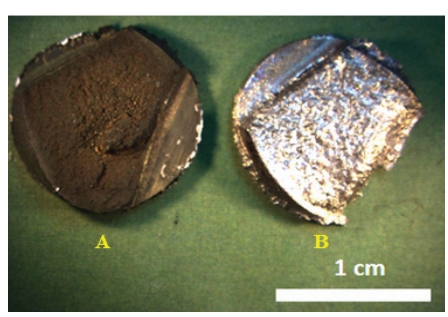

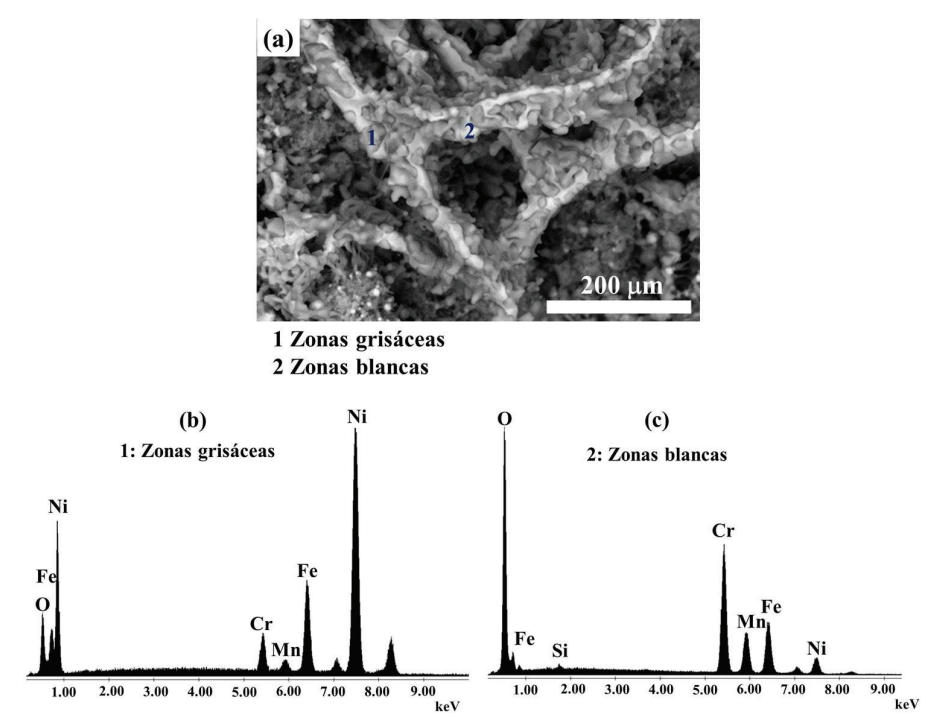

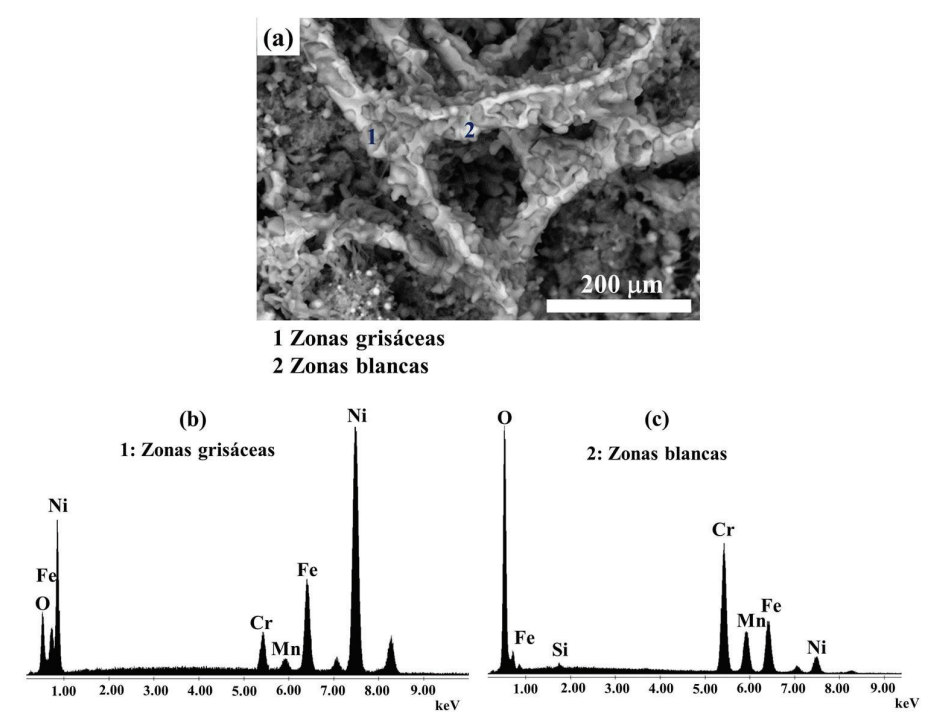

yores a $1 \mu \mathrm{m}$, así como partículas de morfologías cuboidal de color blanco y grises. Cabe mencionar que la formación de estas partículas crecen a partir de la nucleación y crecimiento de las nanopartículas de níquel sobre defectos y el mismo substrato que está compuesto principalmente de $\mathrm{Cr}, \mathrm{Fe}$ y Ni. Básicamente, las partículas de aspecto cuboidal (zona 1) tienen un aspecto laminar con una composición química rica en $\mathrm{Ni}$ con $\mathrm{Fe}$ y Cr, principalmente (Figuras $8 \mathrm{~b}$ y c). Los análisis químicos puntuales sobre la matriz del recubrimiento (zona 2) por EDX muestran picos intensos de níquel (Ni) y picos de mediana intensidad de los elementos químicos de hierro $(\mathrm{Fe})$ y cromo $(\mathrm{Cr})$, así como picos de menor intensidad de carbono (C), molibdeno (Mo) y manganeso $(\mathrm{Mn})$ que corresponden a la composición química del substrato y el carbono proveniente de la etapa de calcinación (Figuras 8b y d).

La Figura 9a muestra un mapeo metalográfico de un corte transversal del recubrimiento en condición de pulido con espesor de entre 50 a $700 \mu \mathrm{m}$ a lo largo de la superficie de fractura. A altas amplificaciones se aprecia la interface (metal base-recubrimiento) y la zona del recubrimiento con una microestructura dendrítica (Figuras 9 b y 9 c). Las microestructuras dendríticas indican la formación de fases líquidas transitorias antes del crecimiento del recubrimiento (Cook y Sorensen, 2011;
Figura 6. Imágenes por estereoscopio de la superficie de fractura después del crecimiento del recubrimiento
Figura 7. a) Imagen de electrones retrodispersados del recubrimiento parcial sobre la fractura de acero inoxidable 304 (muestra A), b) y c) elementos presentes en las zonas grisáceas (1) y blancas (2) identificados por EDX 

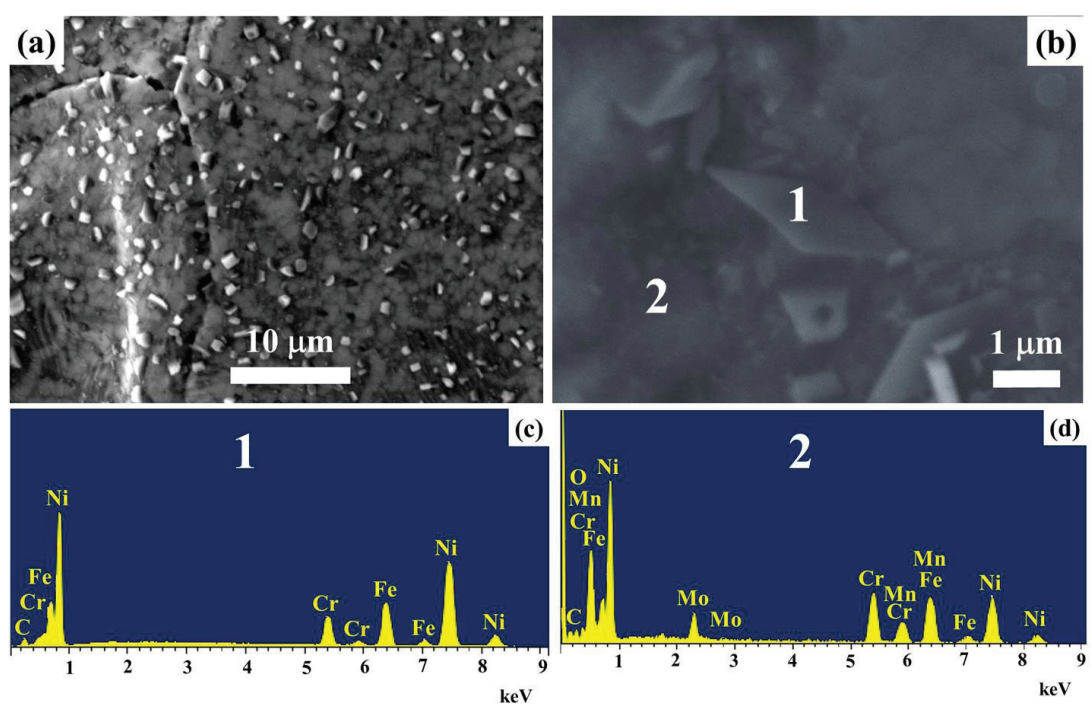

(a)

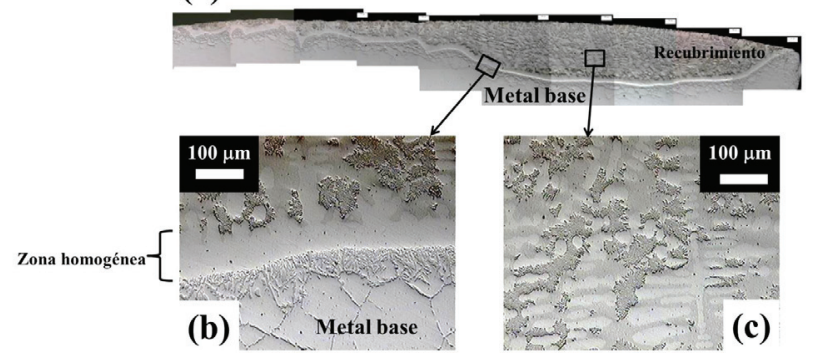

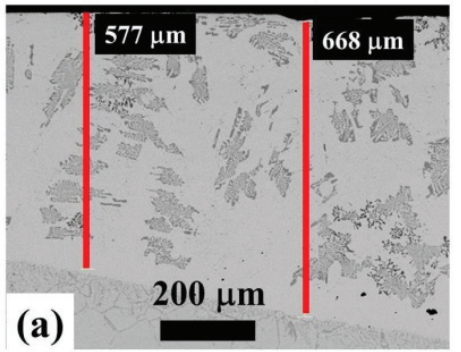

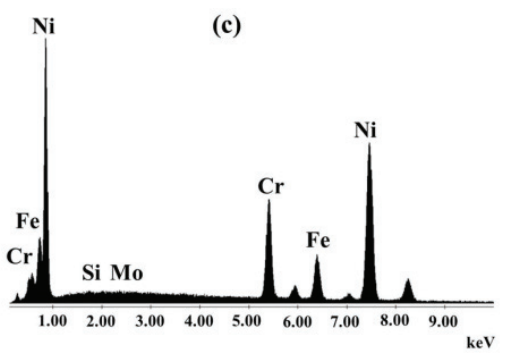

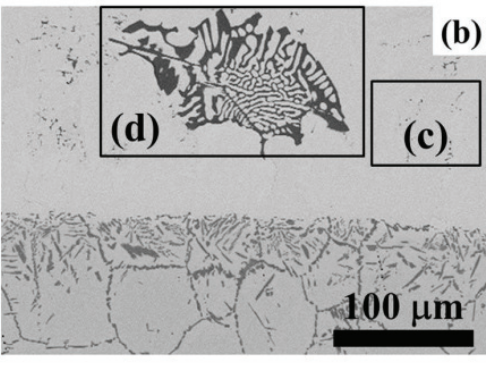

(d)

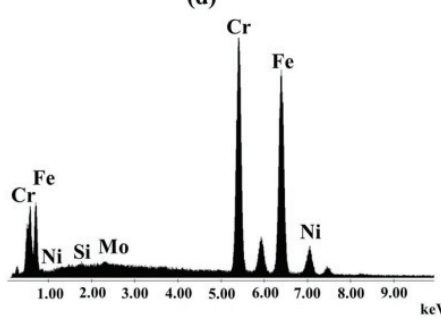

Figura 9. Micrografías de un corte transversal en condición de pulido del recubrimiento obtenido en la muestra $\mathrm{B}$ tratada a $1000^{\circ} \mathrm{C}$ por $60 \mathrm{~min}$, a) mapeo metalográfico del recubrimiento, b) interface entre el recubrimiento-metal base, c) microestructura dendrítica en el recubrimiento

Figura 10. a) y b) Imagen de electrones retrodispersados del recubrimiento y fase laminar de la muestra B tratada a $1000^{\circ} \mathrm{C}$ por $60 \mathrm{~min}, \mathrm{c})$ y d) espectrogramas por EDX de la matriz y fase laminar 
Kerr y Kurz,1996). Se postula que en la superficie se tiene la fase líquida en contacto con el metal base y, se transfiere calor del sólido al líquido (gradiente térmico negativo) e inicia la transferencia de especies químicas a la fase líquida, que permiten por nucleación, formar mayor fracción sólida. Cabe mencionar que en la etapa de la difusión se forma un volumen de líquido entre la intercara del metal base, obteniéndose una composición química diferente a la inicial. Por lo tanto, se forman dos regiones con diferentes concentraciones de soluto disueltos en la fase líquida que aumenta en espesor, mientras la intercara sólida va disminuyendo. En consecuencia, con el tiempo se obtendrá una única fase líquida de composición rica en $\mathrm{Ni}\left(\mathrm{C}_{\mathrm{L}}\right)$ con sus respectivos gradientes de concentración de $\mathrm{Fe}, \mathrm{Cr}$ y Mn, principalmente. Por lo tanto, una vez que la capa líquida está en contacto con el metal base, inicia una difusión rápida de los elementos que difunden del líquido al sólido y los del metal base, hacía el líquido, hasta alcanzar un gradiente de concentración homogéneo en la fase líquida. La tasa de transferencia de especies químicas es una función de los gradientes de concentración en ambas capas. En cuanto inicia la solidificación, la difusión toma un proceso lento y la homogeneización química depende del tiempo, en este trabajo de investigación la zona homogénea es $100 \mu \mathrm{m}$ (Figura 9b).

En otra sección del perfil transversal del recubrimiento de la muestra B atacada electroquímicamente, se muestran microestructuras eutécticas en forma de estructura laminar (Figuras 10a y b). Además, se aprecia una interface entre el metal base y el recubrimiento con precipitados. La composición química cualitativa de esta sección transversal, muestra por espectrogramas de área la composición química de elementos que evidencia que el recubrimiento es rico en $\mathrm{Ni}$ y las fases de aspecto laminar se componen principalmente de $\mathrm{Ni}$, $\mathrm{Fe} \mathrm{y} \mathrm{Cr}$ (ver Figuras 10c y d). En la literatura se reporta que las fases de morfología laminar crecen a partir de reacciones invariantes eutécticas o peritécticas del sistema Ni-Cr-Fe (Ares et al., 2005; Rajasekhar et al., 1997; Kerr y Kurz, 1996).

\section{Conclusiones}

En el marco de estudio de este trabajo de investigación se concluye lo siguiente:

Con base en los resultados obtenidos por DSC se determinó que el etilenglicol es el precursor adecuado para la preparación del gel, debido a su menor temperatura de estabilización. Por otro lado, las etapas del método Pechini tienen la versatilidad de penetrar el $\mathrm{Ni}$ en el interior de las microfisuras y microporos para sellarlas y taparlas en la etapa de la formación del recubrimiento sobre las fracturas de acero inoxidable 304. El recubrimiento obtenido a $1000^{\circ} \mathrm{C}$ por $60 \mathrm{~min}$ muestra una microestructura dendrítica lo que evidencia la formación de una fase líquida transitoria. Por otro lado, este método permite realizar un recubrimiento rico en $\mathrm{Ni}$ sobre superficies que hayan sido fracturadas y retardar o evitar la exposición del metal a un mayor grado de oxidación o corrosión. No obstante, se postula que el recubrimiento servirá como un substrato para la deposición de grafeno, generando en conjunto una película de ultra-alta dureza que será utilizada para incrementar las propiedades tribológicas del acero inoxidable 304.

\section{RefERENCIAS}

Ares, A.E., Caram, R., Jaime, M.A., Ferrandini, P., Dutra, A.T., Gueijman, S.F., Schvezov C. E. (2005). Solidificación y microestructura de un acero inoxidable austenítico. Anales AFA, $17,240-249$.

Blackman, J.A. (2009). Handbook of metal physics, Metallic Nanoparticles, Elsevier, pp. 114-135.

Chen, J., Li, J., Xionga, D., Hea, Y., Jia, Y., Qin, Y. (2016). Preparation and tribological behavior of Ni-graphene composite coating under room temperature. Appl. Surf. Sci., 361, 49-56.

Cook III G.O. y Sorensen C.D. (2011) Overview of transient liquid phase and partial transient liquid phase bonding. J. Mater. Sci., 46, 5305-5323.

Fabian, F.A., Pedra, P.P., Filho, J.L., Duque, J.G., Meneses, C.T. (2015). Synthesis and characterization of $\mathrm{La}(\mathrm{Cr}, \mathrm{Fe}, \mathrm{Mn}) \mathrm{O}_{3}$ nanoparticles obtained by co-precipitation method. Journal of Magnetism and Magnetic Materials, 379, 80-83.

Galceran, M., Pujol, M.C., Aguiló, M., Díaz, F. (2007). Sol-gel modified Pechini method for obtaining nanocrystalline $\mathrm{KRE}\left(\mathrm{WO}_{4}\right)_{2}(\mathrm{RE}=\mathrm{Gd}$ and $\mathrm{Yb})$. Journal of SOL-GEL Science and Technology, 42, 79-88.

Johnston, R.L. (2002). Atomic and Molecular Clusters, Edited Taylor \& Francis, Nueva York, pp. 3-33.

Kerr, H.W. y Kurz, W. (1996). Solidification of Peritectic Alloys. Int. Materials Reviews, 41(4), 129-163.

Marulanda, J.L. y Trujillo, G. (2007). Recovery of wearing pieces with protective coatings. Scientia et Technica, 37, 199-204.

Mota, M.L., Osuna, J.G., Navarro, D., Carrillo, A. (2013). Desarrollo de sustratos trasparentes a base de óxido de lantano por el método de Pechini. Revista Iberoamericana de Polímeros, 14(1), 50-57.

Niederberger, M. (2007). Nonaqueous Sol-Gel Routes to Metal Oxide Nanoparticle. Accounts of Chemical Research, 40, 793-800.

Pechini, M.P. (1967). US Patent No. 3,330,697, 1-7.

Puente, B.A., García L.A., De León E.L., López M.G. (2012). Nanoparticulas de $\mathrm{Ni} / \mathrm{NiO}$ y $\mathrm{Cu}-\mathrm{Ag}$ obtenidas mediante el método 
de Pechini: Síntesis y caracterización. Superficies y Vacio, 25(3), 183-187.

Raba, A., Suarez, D.N., Martinez, J.J., Rojas, H.A., Rincón, M. (2015). Pechini method used in the obtention of semiconductor nanoparticles based niobium. DYNA Rev. Fac. Nac. Minas, 82 (189), 52-58.

Rajasekhar, K., Harendranath, C.S., Raman, R., Kulkarni, S.D. (1997). Microstructural evolution during solidification of austenitic stainless weld metals: a color metallographic and electron microprobe analysis study. Materials Characterization, 38, 53-65.

Rao, C.N.R., Müller, A, Cheetham, A.K. (2004). The chemistry of nanomaterials: synthesis, properties and applications, 1(2), Edited by WILEY-VCH, Verlag GmbH \& Co. KGaA, Weinheim, USA, 1-27.

Sahoo, C.K. y Masanta, M. (2017). Microstructure and mechanical properties of TiC-Ni coating on AISI 304 steel produced by TIG cladding process. J. Mater. Proces. Techn., 240, 2017, 126-137.
Solarte, N.J., Ramírez, A.E., Villaquirán, C., Tirado, L., Gaona, S. (2015). Síntesis de polvos cerámicos de hexaferrita de estroncio por los métodos pechini y combustión. Revista. Latinoamericana de Metalúrgia y Materiales, 35(2), 276-284.

Vaqueiro, P. y López, M.A. (1998). Synthesis of yttrium aluminium garnet by the citrate gel process. Journal of Material Chemistry, 8(1), 161-163.

Wang; D. y Bierwagen, P. (2009). Sol-gel coatings on metals for corrosion protection. Progress in Organic Coatings, 64, 327-338.

Xiaoming, L. Xuejiao, M., Dalu, S., Babar, S., Yang. Q. (2015). Crystalline characteristics and superconducting properties of Bi2212 thin films prepared by the Pechini sol-gel method: the effect of different substrates on the film growth. Journal of Applied Crystallography, 48, 37-43.

Zaki, T. y Kabel, K.I. (2012). Hassan H. Using modified Pechini method to synthesize $\alpha-\mathrm{Al}_{2} \mathrm{O}_{3}$ nanoparticles of high surface area. Ceramics International, 38, 4861-4866. 